\title{
AMORPHOUS Bi-Ge-S(Se) SYSTEMS STUDIED BY X-RAY Ge $K$ EMISSION AND ABSORPTION BANDS
}

\author{
J. Drahokoupil, I. Drbohlav, M. PolC̆ıK \\ Institute of Physics, Czech Academy of Sciences \\ Cukrovarnická 10, 16253 Praha 6, Czech Republic
}

AND L. TICHÝ

University of Chemical Technology, 53210 Pardubice, Czech Republic

The X-ray Ge $K$ absorption (emission) bands were measured in the stoichiometric glass system $\left(\mathrm{GeS}_{2}\right)_{x}\left(\mathrm{Bi}_{2} \mathrm{~S}_{3}\right)_{1-x}(x=0 \div 0.5)$ and in the $\mathrm{Ge}_{20} \mathrm{Bi}_{y} \mathrm{Se}_{80-y}$ system $(y=0,7$ and 13$)$. The results do not confirm the existence of the $\mathrm{Ge}-\mathrm{Bi}$ bond and they imply that the $\mathrm{GeS}_{2}$-clusters are formed in both studied systems.

PACS numbers: $78.70 . \mathrm{Dm}$

\section{Introduction}

The Ge-Bi-S(Se) glasses prepared by quenching from the melt have been studied because they reveal, in contrast to most other chalcogenide glasses, the $n$-type conductivity at higher concentrations of Bi. There has been a long discussion about the structure of these materials, particularly about the existence of the $\mathrm{Ge}-\mathrm{Bi}$ bond. Its existence is supposed in the $\mathrm{Ge}-\mathrm{Bi}-\mathrm{S}(\mathrm{Se})$ system in [1-3]. On the contrary, Elliot and Steel [4], who measured the extended X-ray absorption fine structure (EXAFS), exclude the existence of the Ge-Bi bond.

We decided to study the X-ray Ge $K$ emission and absorption bands because of their sensitivity to the short range order. The $K$-emission bands correspond to the transition of the Ge $1 s$ hole to the valence band states of $p$-symmetry and the $K$ absorption bands (represented by the absorption coefficient $\mu(E)$ ) correspond to the transition of the $1 s$ electron to the conduction band $p$-states. In both cases the symmetry is related to the Ge atoms. Due to the localization of the $1 s$ state the X-ray emission and absorption bands sensitively reflect the changes in the first coordination sphere of the Ge atom as to its symmetry and/or the kind of atoms. 


\section{Experiment}

The X-ray bands were performed by two-crystal spectrometers with silicon single crystals and X-ray spectrometry tubes. The Ge $K_{\beta_{2}}$ emission bands were measured in the $(m,-n)$ position using $\mathrm{Si}(220)$ and $\mathrm{Si}(400)$. The fluorescence method of excitation was used [5].

The Ge $K$ absorption bands were measured in the $(m, n)$ position using the $\mathrm{Si}(220), \mathrm{Si}(111)$ and $\mathrm{Si}(220), \mathrm{Si}(220)$ crystal for $\mathrm{Ge}-\mathrm{Bi}-\mathrm{S}$ and $\mathrm{Ge}-\mathrm{Bi}-\mathrm{Se}$ systems respectively (for experimental details see [6]).

The glasses were prepared by direct synthesis from the corresponding elements by the conventional melt quenching technique $[6,7]$. The glassy nature of the prepared samples was tested by X-ray diffraction measurements, infrared microscopy (100x magnification) and differential thermal analysis.

Figures $1 \div 3$ show the measurements. Each point represents an average of several repeated runs. As reference energies, the Ge $K_{\beta_{1,3}}$ line of pure germanium (emission) and the $\mathrm{W} L_{\gamma_{1}}$ line (absorption) were used.

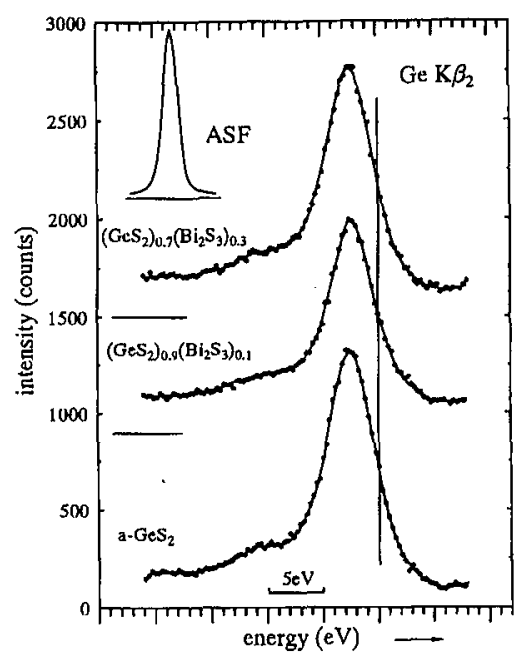

Fig. 1. The measured X-ray Ge $K$ emission bands of the Ge-Bi-S system. ASF is the apparatus smearing function.

\section{Results}

The measured Ge $K_{\beta_{2}}$ emission bands of the $\mathrm{Ge}-\mathrm{Bi}-\mathrm{S}$ system are shown in Fig. 1. The apparatus srrearing function (ASF) gives the resolution of the spectrometer. The absolute energy scale is not given because it is not important for the interpretation; furthermore the precision of the tabulated reference line is of the order of $1 \mathrm{eV}$. Nevertheless, it seems informative to know the energy region of the spectra; the tabulated values [8] of pure germanium are $11100.8 \mathrm{eV}$ for $\mathrm{Ge}$ $K_{\beta_{2}}$ and $11103.6 \mathrm{eV}$ for the $\mathrm{Ge} K$ absorption edge. 


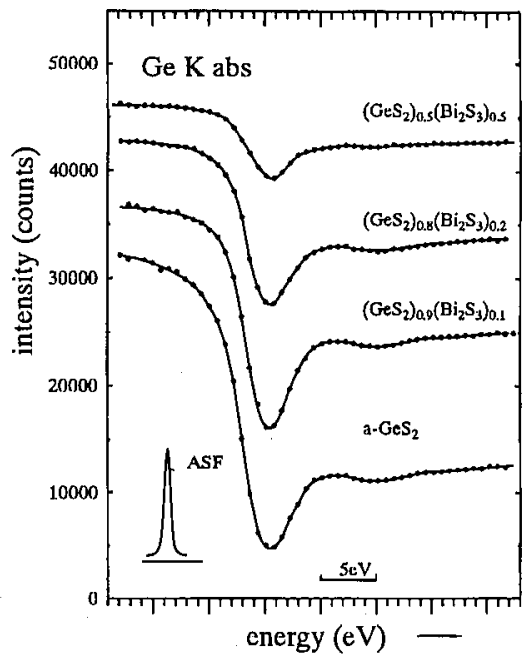

Fig. 2. The measured absorption curves $N(E)$ of the Ge-Bi-S system. Each measurement was monitored to $N_{0}(E)=50000$ counts. The absorption coefficient $\mu$ is proportional to $\ln \left[N_{0}(E) / N(E)\right]$. ASF is the apparatus smearing function.

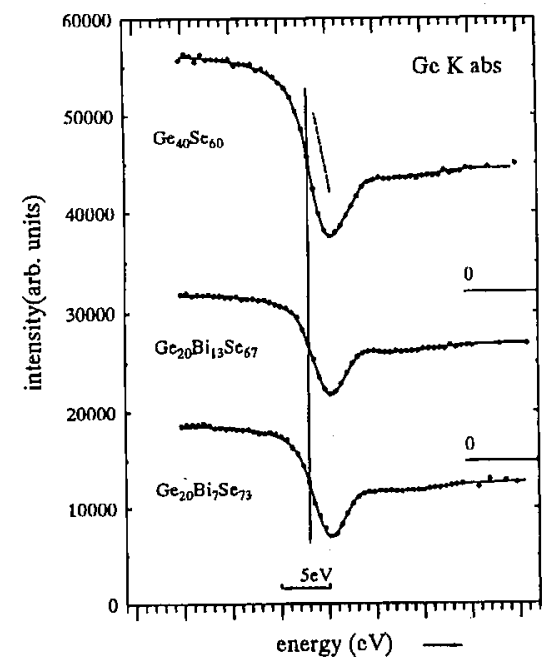

Fig. 3. The measured absorption curves $N(E)$ of the Ge-Gi-Se system. The conditions of the measurement are the same as in Fig. 2. The dashed line near the $\mathrm{Ge}_{40} \mathrm{Se}_{60}$ curve schematically indicates the shift of the edge found by Agnihotri et al. [3].

The energy and shape of the emission band do not depend on the Bi content (see vertical line in Fig. 1).

The Ge $K$ absorption bands of the $\mathrm{Ge}-\mathrm{Bi}-\mathrm{S}$ and $\mathrm{Ge}-\mathrm{Bi}-\mathrm{Se}$ systems are shown in Figs. 4 and 5 . They represent the energy dependence of the absorption coefficient 


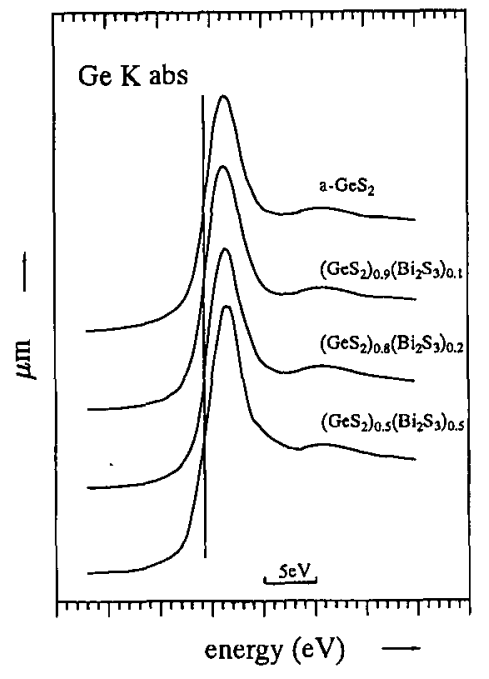

Fig. 4. The X-ray Ge $K$ absorption bands of the stoichiometric Ge-Bi-S system; $\mu(\mathrm{E})$ is the absorption coefficient.

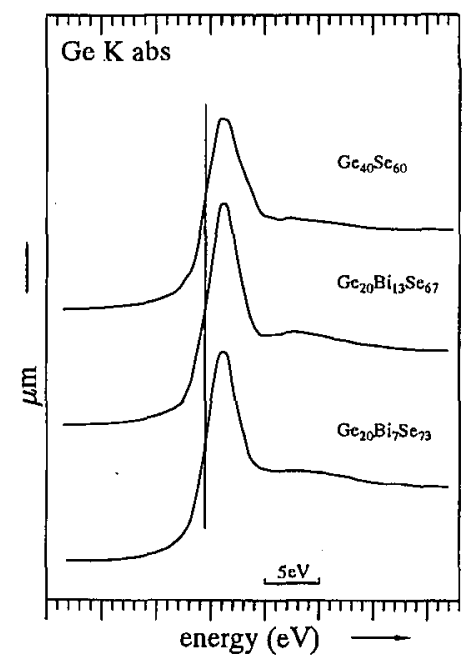

Fig. 5. The X-ray Ge $K$ absorption bands of the Ge-Bi-Se system; $\mu(E)$ is the absorption coefficient.

$\mu m$ ( $\mu$ is the absorption coefficient, $m$ is the mass of the sarnple per $\mathrm{cm}^{2}$ ). The measured curves were corrected, which means that they were deconvoluted by the apparatus smearing function. Again, we see practically no changes in the energy of the absorption edge or the band shape.

The studied non-stoichiometric glasses are more often specified as $\mathrm{Ge}_{x} \mathrm{Bi}_{y} \mathrm{~S}(\mathrm{e})_{1-x-y}$. Using this notation we give the composition of our $\mathrm{Ge}-\mathrm{Bi}-\mathrm{S}$ samples in Table for corriparison. 


\section{TABLE}

The $\mathrm{Ge}_{z} \mathrm{Bi}_{y} \mathrm{X}_{1-x-y}$ notation for the samples measured $(X=S$ or $\mathrm{Se})$.

\begin{tabular}{l|l}
\hline$\left(\mathrm{GeX}_{2}\right)_{0.9}\left(\mathrm{Bi}_{2} \mathrm{X}_{3}\right)_{0.1}$ & $\mathrm{Ge}_{0.28} \mathrm{Bi}_{0.06} \mathrm{X}_{0.66}$ \\
$\left(\mathrm{GeX}_{2}\right)_{0.8}\left(\mathrm{Bi}_{2} \mathrm{X}_{3}\right)_{0.2}$ & $\mathrm{Ge}_{0.23} \mathrm{Bi}_{0.12} \mathrm{X}_{0.65}$ \\
$\left(\mathrm{GeX}_{2}\right)_{0.7}\left(\mathrm{Bi}_{2} \mathrm{X}_{3}\right)_{0.3}$ & $\mathrm{Ge}_{0.19} \mathrm{Bi}_{0.17} \mathrm{X}_{0.64}$ \\
$\left(\mathrm{GeX}_{2}\right)_{0.5}\left(\mathrm{Bi}_{2} \mathrm{X}_{3}\right)_{0.5}$ & $\mathrm{Ge}_{0.12} \mathrm{Bi}_{0.25} \mathrm{X}_{0.63}$
\end{tabular}

\section{Discussion}

Our measurentents show no changes in the Ge $K$ bands dependent on the $\mathrm{Bi}$ concentration, which means that the coordination around Ge-atoms does not change as well. The formation of the $\mathrm{Ge}-\mathrm{Bi}$ bond would bring about a significant change in the $\mathrm{X}$-ray bands because the $\mathrm{Bi}$-atoms are unlike in valency and in requirements for the syrrmetry of neighbours in comparison with sulphur or selenium (the sensitivity of the Ge $K$ absorption bands can be demonstrated e.g. by the significant difference in their shapes going from amorphous GeS to the crystalline GeS [6]).

Our results do not agree with those presented by Agnihotri et al. [3] who measured the Ge $K$ absorption edges in Se rich Ge-Bi-Se glasses. They found a shift of the absorption edge of $1.91 \mathrm{eV}$ for the $\mathrm{Ge}_{22} \mathrm{Bi}_{10} \mathrm{Se}_{68}$ glass in comparison with the $\mathrm{Ge}_{22} \mathrm{Se}_{78}$, from which they deduced the presence of the $\mathrm{Ge}-\mathrm{Bi}$ bond. However, it is necessary to remark that in comparison with the two crystal spectrometers the apparatus used by Agnihotri et al. has a very low resolving power and the photographic registration of the spectra. From the $\mathrm{Ge} K$ absorption edge measurements by Zhou et al. [9] it follows that the position of the absorption edge of $\mathrm{Ge}_{40} \mathrm{Se}_{60}$ and $\mathrm{Ge}_{20} \mathrm{Se}_{80}$ is practically the same. For the Ge-Bi-S system we studied the stoichiometric glasses and so it can be expected that more $\mathrm{Ge}-\mathrm{Bi}$ bonds are formed than in systerns rich in $\mathrm{S}(\mathrm{Se})$. Yet our results do not support it. Thus we conclude that the changes induced by doping $\mathrm{GeS}_{2}$ with $\mathrm{Bi}$ do not concern the nearest neighborhood of germanium atorns. We obtained similar results for the Sb-doped systern [6].

For the stoichiornetric Ge-Bi-S system and the concentration range studied by us the measurements of the optical gap [10] give changes up to $2 \mathrm{eV}$ correlated with the Bi-content. Our measurements show no shift in the emission band edge (see vertical line in Fig. 1) or the absorption edge (Fig. 5). Therefore we can infer that the gap width corresponds to that in amorphous $\mathrm{GeS}_{2}{ }^{*}$.

\footnotetext{
*It should be emphasized that the Ge $K$ bands yield information about the $p$-partial density of states (PDOS) around Ge-atoms ( $p$-states of germanium) and it is very unlike that any change in the position of the valence band maximum and conduction band minimum would not be accompanied by the change of the $p$-symmetry PDOS.

The disagreement between our measurements and the measurements of the optical gap can be interpreted by means of the idea of clusters of $\mathrm{GeS}_{2}$ and $\mathrm{Bi}_{2} \mathrm{~S}_{3}$ that the Ge-Bi-S system consists of. The optical properties of the $\mathrm{Ge}-\mathrm{Bi}-\mathrm{S}$ system can then be explained by means of the percolation theory as proposed in [10].
} 
Considering the fact that the X-ray bands describe the states localized on the germanium atoms our measurement support the idea that the glasses in question are built of $\mathrm{GeS}_{4}\left(\mathrm{GeSe}_{4}\right)$ clusters.

\section{Conclusions}

The X-ray Ge $K$ absorption bands were measured in the Se-rich Ge-Bi-Se system and the Ge $K$ emission and absorption bands were measured in the stoichiometric $\mathrm{Ge}-\mathrm{Bi}-\mathrm{S}$ system. Our measurements support the idea of forming the microclusters of $\mathrm{GeS}_{2}$ in these materials and do not confirm the existence of $\mathrm{Ge}-\mathrm{Bi}$ bonds, in particular the dependence of the such bonds on the concentration of $\mathrm{Bi}$.

\section{Acknowledgments}

This work was supported by grants of the Grant Agency of the Czechoslovak Academy of Sciences Nos. 42-901 and 11-076 and by the grant of the Grant Agency of the Czech Republic No. 202/94/0669.

\section{References}

[1] K.L. Bhatia, D.P. Gosain, V.K. Bhatnagar, Phys. Rev. B 35, 4503 (1987).

[2] A.K. Sharma, K.L. Bhatia, V.K. Bhatnagar, S.K. Malik, W. Kratschmer, J. Non-Cryst. Solids 108, 309 (1989).

[3] A.K. Agnihotri, A. Kumar, A.N. Nigam, J. Non-Cryst. Solids 93, 267 (1987).

[4] S.R. Elliot, A.T. Steel, Phys. Rev. Lett. 57, 1316 (1986).

[5] J. Drahokoupil, A. Fingerland, Czech. J. Phys. B 18, 1190 (1968).

[6] J: Drahokoupil, I. Drbohlav, J. Chval, L. Tichý, M. Polčík, J. Phys,, Condens. Matter 5, 5997 (1993).

[7] L. Tichý, H. Tichá, A. Pačesová, J. Petzlt, J. Non.-Cryst. Solids 128, 191 (1991).

[8] J.A. Bearden, X-ray Wavelengths, US Atomic Energy Commission, Oak Ridge 1964.

[9] W. Zhou, M. Paesler, D.E. Sayers, Phys. Rev B 43, 2315 (1991).

[10] P. Nagels, L. Tichý, H. Tichá, A. Tř́ska, Physics of Disordered Materials, Ed. D. Adler, H. Fritzsche, R. Ovshinsky, Plenum, New York 1985, p. 645. 